\title{
Neuropsychological deficits, syndromes, and cognitive competency in schizophrenia
}

\author{
Karin M. Christensen and Catherine A. Mateer \\ University of Victoria, Victoria, Canada \\ Richard Williams \\ Eric Martin Pavilion, Victoria, Canada \\ Todd S. Woodward \\ University of Victoria, Victoria, Canada
}

\begin{abstract}
Introduction. Neuropsychological functioning connects neuropathology and symptoms in schizophrenia. Previous work suggests that deficient initiation and inhibition underlie the psychomotor poverty and disorganisation syndromes, respectively. Furthermore, although the syndromes are associated with impairments in everyday functioning, cognitive competency $(\mathrm{CC}$; cognitive skills for independent living) has been neglected as an outcome. This study tested a threelevel model of schizophrenia pathology (Neuropsychological dysfunction $\rightarrow$ Syndromes $\rightarrow$ CC), using unstructured neuropsychological tasks to measure initiation and inhibition.

Methods. Participants were 40 adults with schizophrenia. A verbal picture description and the Tinkertoy test yielded initiation and inhibition measures with good interrater reliability. Symptoms were rated using the SANS and SAPS, and an insight scale was administered. The Cognitive Competency Test utilised simulated situations to assess CC.

Results. Initiation failed to predict psychomotor poverty, but affected CC directly. Only one indicator of disinhibition (intermingling of personal material into speech) predicted disorganisation, which, through mediation, led to $\mathrm{CC}$ deficits. Insight
\end{abstract}

Correspondence should be addressed to Dr. Karin Christensen, G. F. Strong Rehab Centre, 4255 Laurel Street, Vancouver, BC, V5Z 2G9, Canada; e-mail: kchriste@vanhosp.bc.ca.

This research was supported by grants to the first author from the Dr. Norma Calder Schizophrenia Foundation, the Robert and Douglas Vickery Graduate Award, Janssen-Ortho Inc., and the Sara Spencer Research Award in Applied Social Sciences.

The authors wish to thank the following individuals for their assistance with this research: Christine Schwartz for scoring of transcripts and videos; Lizanne Walsh and Jasmine Sharp for support in data collection from clinical settings; Dr Ross Norman for consultation regarding syndrome scoring; Dr Peter Liddle for expert comments on cognition in schizophrenia syndromes; Drs Mike Hunter, Holly Tuokko, Roger Graves, and Rennie Warburton for valuable comments throughout the project; and Dr Elton Ngan for helpful advice on an early draft of the manuscript.

(C) 2005 Psychology Press Ltd 
correlated with disorganisation and contributed to CC. Unique effects of initiation, disorganisation, and insight, combined, explained $58 \%$ of $\mathrm{CC}$ variance.

Conclusions. Partial support for the three-level model was obtained. Specific neuropsychological abilities and symptoms explain a substantial proportion of the variance in cognitive competency.

Schizophrenia is a widespread and devastating mental illness. A diagnosis of this condition usually implies deterioration in social, occupational, and intellectual functioning. Over the decades of research in schizophrenia, a broad spectrum of its facets has been examined, ranging from the genetic and cellular levels to the outcome in terms of daily functioning. However, much remains to be understood about the neuropsychological foundations of symptoms, and the ways in which these aspects of the illness impact everyday functioning. Thus, there is a need for research that connects various discrete aspects of schizophrenia, to allow for more comprehensive appreciation of the illness.

Some authors have argued that the pathology in schizophrenia can best be understood through the interaction of abnormalities at various levels. This concept is referred to as the levels of understanding model (see Frith, 1992, p. 13; Mortimer, 1994; Mortimer, 1992) and can be depicted "Specific brain pathologies $\rightarrow$ Neuropsychological dysfunction $\rightarrow$ clinical phenomena". The neuropsychological level connects neuropathology and observable symptoms, and is an important link between two more commonly investigated aspects. The levels of understanding model further advocates for a focus on symptoms, as opposed to the study of the more diverse and fluctuating diagnostic category "schizophrenia".

The neuropsychology of the three schizophrenia syndromes has been elucidated in recent years. Substantial evidence suggests that the psychomotor poverty syndrome (i.e., poverty of speech, blunted affect, and decreased spontaneous movement) is associated with impaired cognitive initiation and generation, whereas the disorganisation syndrome (inappropriate affect and formal thought disorder) is related to deficient inhibition of inappropriate responses (Allen, Liddle, \& Frith, 1993; Frith, Leary, Cahill, \& Johnstone, 1991; Hammer, Katsanis, \& Iacono, 1995; Liddle \& Morris, 1991). While its cognitive underpinnings are less clear, the reality distortion syndrome (delusions and hallucinations) may be related to deficient self-monitoring of thoughts and actions (Frith, 1992; Frith \& Done, 1988, 1989; Mlakar, Jensterle, \& Frith, 1994).

In addition to these clinical syndromes, unawareness of illness is particularly severe and pervasive in schizophrenia as compared to other psychiatric disorders, and is unique to schizophrenia among the psychoses (Amador et al., 1994; Amador \& Kronengold, 1998; Amador, Strauss, Yale, \& Gorman, 1991). Lack of insight describes a self-percept that is discrepant from that of the community (Amador et al., 1991). In schizophrenia the basic picture involves lack of awareness of associated deficits, consequences, and treatment need 
(Amador et al., 1991). The available literature suggests that poor insight may relate to the executive cognitive deficits in schizophrenia, purportedly caused by prefrontal dysfunction (Lysaker \& Bell, 1994, 1995; Young, Davila, \& Scher, 1993).

There is evidence that the observable manifestations of schizophrenia lead to impairments in everyday functioning. For example, disorganisation syndrome negatively affects life skills (Liddle, 1987a), and poor insight is associated with psychosocial (Amador et al., 1994) and work-related problems (Lysaker \& Bell, 1995), as well as treatment noncompliance (Amador et al., 1991). Based on these observations, the causal pathways described above can be extended to the level of cognitive functioning in daily life, or cognitive competency. Thus, "Specific brain pathologies $\rightarrow$ Neuropsychological dysfunction $\rightarrow$ Clinical phenomena $\rightarrow$ Cognitive competency". Though clearly important, cognitive competency has been given little attention.

The goal of the present study was to test the last three levels in the model above. We endeavoured to link neuropsychology to syndromes, and syndromes to cognitive competency, the latter representing a critical outcome. We used standard measures for the syndromes and for cognitive competency, but innovative measures for the neuropsychological level. Prior research in diverse brain-damaged populations demonstrates that open-ended and self-directed (i.e., unstructured) tasks are remarkably sensitive measures of behavioural initiation, maintenance, and inhibition, and are useful in discriminating patient groups (Bayless, Varney, \& Roberts, 1989; Lezak, 1982; Martzke, Swan, \& Varney, 1991; Mendez \& Ashla-Mendez, 1991). These studies used tasks such as the Tinkertoy test (Lezak, 1982) and verbal descriptions of pictures. Therefore, we aimed to utilise measures developed from similar tasks - one verbal and one nonverbal - to elicit deficits in initiation and inhibition. The reality distortion syndrome was not investigated, because: (a) its cognitive underpinnings are unclear (Liddle \& Morris, 1991; Moritz et al., 2001; Woodward, Ruff, Thornton, Moritz, \& Liddle, 2003; Woodward, Thornton, Ruff, Moritz, \& Liddle, 2004); and (b) there is no evidence that it predicts everyday functioning (Green, 1998, pp. 154-155; Liddle, 2001, pp. 107 \& 125).

In summary, our first aim was to relate neuropsychological performance to the psychomotor poverty and disorganisation syndromes, using unstructured neuropsychological tasks. We hypothesised (a) that deficient initiation of selfgenerated thoughts and actions underlies the psychomotor poverty syndrome; and (b) that deficient inhibition of inappropriate responses causes the disorganisation syndrome. Our second aim was to examine the impact of these cognitive and behavioural abnormalities on overall cognitive competency. Thus, we predicted that both syndromes cause impaired cognitive competency, and that they mediate the effects of cognitive deficits on cognitive competency. Figure 1 depicts the hypothesised relationships. Our third objective was to explore the relation of unawareness of illness to the above variables. 


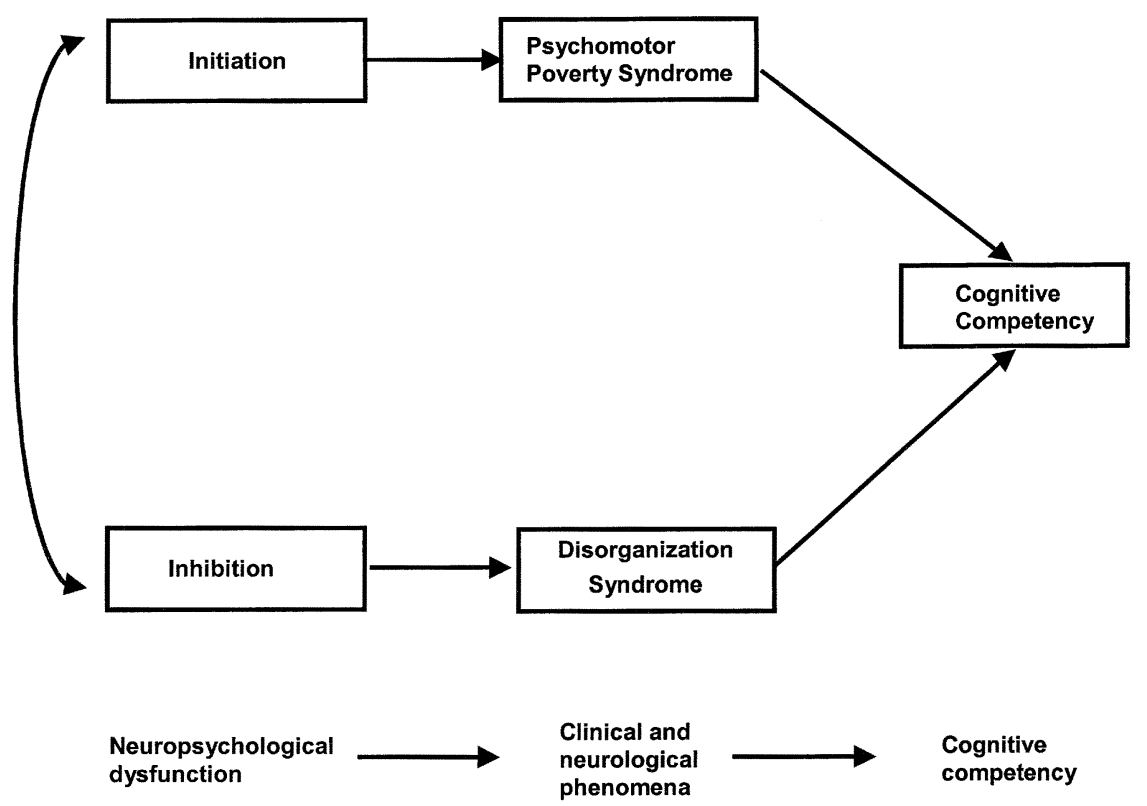

Figure 1. This figure represents the relationships hypothesised. The flow of causality runs from neuropsychology to syndromes, and syndromes to cognitive competency. Deficient initiation was expected to underlie the psychomotor poverty syndrome; deficient inhibition was expected to cause the disorganisation syndrome. Both syndromes were hypothesised to predict impaired cognitive competency, thus mediating the effects of neuropsychological deficits on cognitive competency.

\section{METHOD}

\section{Participants}

Participants were 40 patients ( 27 male and 13 female) with a current diagnosis of schizophrenia according to DSM-IV criteria (American Psychiatric Association, 1994). Diagnoses were confirmed by author R.W. Participants were receiving psychiatric treatment (by R.W., with one exception) at either the Eric Martin Pavilion of the Royal Jubilee Hospital (in Victoria, BC, Canada) or the Victoria Mental Health Centre. Potential participants were excluded if they had another Axis I disorder concurrently, or a history of neurological disease or head injury.

Participants ranged in age from 17 to 51 years (mean 30.75), and had a mean education of 11.65 years. Premorbid verbal intellectual ability was estimated using the Quick Test (Ammons \& Ammons, 1962), and the sample mean was 98.28. There were 11 inpatients and 29 outpatients; mean duration of illness (i.e., time since diagnosis) was 6.95 years. All participants were taking antipsychotic medications at the time of assessment, and 10 were taking anti- 
cholinergics in addition. Participants were judged to be clinically stable by the treating psychiatrist.

Recruiting was accomplished by providing suitable patients with written study information and allowing them to state whether they gave permission to be contacted at a later date, at which time they could accept or decline participation. Informed consent was obtained at the time of testing.

\section{Procedures and materials}

Medication data, symptom ratings, and psychometric data were gathered within one day of each other. Symptom ratings were completed by R.W. The unstructured neuropsychological tasks, Cognitive Competency Test, and insight rating were administered by K.C. Within the session, the unstructured tasks were randomly assigned to first or last position relative to other tests, to control for potential effects of initial test anxiety versus later decreased inhibition. Testing required approximately 1.5 hours.

Verbal unstructured task. This task involved telling a story in response to a picture of a mammoth hunt scene, hereafter referred to as "MHP". (The stimulus was from the Test of Written Language: 2; Hammill \& Larsen, 1988.) This picture was chosen over other potential stimuli because it is more complex, and hence may elicit more speech.

Participants were instructed as follows: "I am going to show you a picture. I want you to look carefully at the whole picture, and then tell me a story about it. Do not point to the picture. Keep talking about the picture until I ask you to stop. Do you understand what to do?" A prompt was given if a participant produced no speech for 30 seconds at any time; this was done only once. If a participant declared that he/she had finished before the time had elapsed, he/she was told: "You have a little more time". Responses were audiotaped for 3 minutes and transcribed. Total number of words and response latency (seconds to first utterance) were used as measures of initiation.

Three types of inappropriate communications were defined for the MHP that are theoretically related to disinhibition. The first, "intermingling", refers to the inappropriate and disruptive blending of personal material into thinking and speech, and has been described as reflecting failure of higher order cognitive control, or executive functioning (Harrow, Lanin-Kettering, Prosen, \& Miller, 1983; Harrow \& Prosen, 1979). The second communication disturbance, "missing information references" (MIRs; Docherty, DeRosa, \& Andreasen, 1996), involves references to information not previously presented and not known by the listener. MIRs may result from a failure to inhibit prepotent "shorthand" references (e.g., she and he), resulting in omission of the necessary background needed by the listener. In this regard, MIRs can be considered similar to the impulsivity observed in the pseudopsychopathic orbital prefrontal 
syndrome (Blumer \& Benson, 1975; Cummings, 1993). Finally, “perseverations" were operationalised as inappropriate repetitions of words, phrases, or ideas, and are also regarded as a reflection of weakened inhibition (Hauser, 1999).

Non-verbal unstructured task. The Tinkertoy test (TT; Lezak, 1982) was used. Tinkertoys are children's constructional play materials consisting of 50 pieces (knobs, wheels, rods, etc.). Instructions were as follows: "Under this cloth are some plastic pieces. When I uncover them, I'd like you to make whatever you want with them. You will have at least 5 minutes, and as much more time as you wish, up to 10 minutes, to make something. Do you understand what to do?" A prompt was given if a participant produced no action for 30 seconds at any time during the task; this was done only once. If a participant declared that he/she had finished before 5 minutes had elapsed, he/she was told "You have a little more time". Performance was videotaped. After 10 minutes, participants were stopped and asked what they had made. As described by Lezak (1982), the number of pieces used and the appropriateness of the construction's name were recorded. If a participant made more than one construction, we required each one to be appropriately named to receive a "yes" score. Response latency (seconds to first piece touched) was also recorded.

Analysis of videotapes provided three measures presumed to reflect disinhibition: the number of "false starts" (touching a piece but not picking it up, or picking up a piece only very briefly); the number of "disconnections" (disconnecting pieces that had been connected); and the number of odd or inappropriate behaviours during the task.

Measures from unstructured tasks. Inhibition capacity was defined as an aggregate of the errors described on the MHP and the TT. (Appropriateness of TT names was excluded due to lack of variation.) To separate output measures from error measures, each error sum was divided by amount of output: MHP errors by the number of words produced, and TT errors by the amount of time on task.

For initiation capacity, we elected not to use a simple aggregate, because the intercorrelations among the initiation measures (i.e., number of words on the MHP task, latency to first utterance, number of TT pieces used, and latency to first piece touched) were fairly low (ranging from .16 to .41), and the initiation aggregate should reflect variance common to these measures. Thus, a weighted aggregate was created using principal component analysis (PCA). The resulting aggregate score emphasises the commonality among the initiation scores, while also including the unique aspects of individual measures. The PCA on the four initiation measures resulted in one component accounting for $44 \%$ of the total variance, with aggregate weights of $-.79, .75,-.43$ and .75 , respectively, as specified by the factor loadings. 
Assessment of cognitive competency. The Cognitive Competency Test was used (CCT; Wang, 1990; Wang \& Ennis, 1986). The CCT assesses basic life skills using paper-and-pencil simulations. It is designed to provide a relatively representative assessment (Wang \& Ennis, 1986), and to minimise the inference between psychological assessment and everyday functioning (Wang, 1990). Cognitive competency as rated by the CCT is not synonymous with mental competency (in the legal sense), and thus, cognitive functions can by examined despite psychotic symptoms which may cause mental incompetence. The CCT consists of eight subtests, covering areas such as completing application forms; reading and interpreting pictured scenes; recalling practical information; managing financial materials; making safety judgements; and using visuospatial memory and orientation skills. For details, see Wang and Ennis (1990) or the CCT Manual (Wang, Ennis, \& Copland, 1987). The Average Total Score (ATS) provides an overall index of cognitive functioning.

Symptom ratings. These were completed by the treating psychiatrist, using the Scale for the Assessment of Negative Symptoms (SANS; Andreasen, 1983) and the Scale for the Assessment of Positive Symptoms (SAPS; Andreasen, 1984). Each item is rated for its severity on a scale from 0 to 5 . The SANS consists of scales measuring alogia, affective blunting, avolition-apathy, anhedonia-asociality, and attentional impairment. The SAPS includes scales for hallucinations, delusions, bizarre behaviour, and positive formal thought disorder. The SANS and SAPS were used because the three schizophrenia syndromes can be derived from them (Liddle, 1987b; Liddle \& Barnes, 1990; Liddle et al., 1992; Malla, Norman, Williamson, Cortese, \& Diaz, 1993).

For this sample, mean $(S D)$ global ratings were as follows: alogia 1.48 (1.32), affective blunting 2.23 (1.19), avolition-apathy 2.8 (.76), anhedonia-asociality 3.43 (1.30), attentional impairment 1.70 (1.30), hallucinations 1.08 (1.42), delusions 1.83 (1.47), bizarre behaviour 1.28 (1.45), and formal thought disorder $2.08(1.29)$.

Syndrome scores were computed by summing the relevant symptom scores (Liddle, 1987b; Liddle \& Barnes, 1990; Liddle et al., 1992; Malla et al., 1993). Psychomotor poverty was computed by summing: (a) the mean of four scores for blunting of affect (affective nonresponsivity, unchanging facial expression, paucity of expressive gestures, and lack of vocal inflections); (b) the score for poverty of speech; and (c) the score for decreased spontaneous movement. Disorganisation was the sum of inappropriate affect, poverty of speech content, and positive formal thought disorder. Because an exploratory factor analysis revealed that poverty of speech content loaded on the psychomotor poverty syndrome in addition to the disorganisation syndrome (Christensen, 1998), the effect of poverty of speech was partialled out of poverty of speech content. This correction of the poverty of speech content item resulted in factor loadings consistent with expectations, and this new measure was used for all analyses 
involving the disorganisation syndrome score. Reality distortion was the sum of voices commenting, persecutory delusions, and delusions of reference.

Insight rating. A brief questionnaire was modelled after the general items from the Scale to assess Unawareness of Mental Disorder (SUMD; Amador \& Strauss, 1990; Amador et al., 1993). The three most common definitions of insight (global awareness of mental disorder, awareness of medication effects, and awareness of social consequences) were assessed, for both current and retrospective insight ("retrospective insight" refers to current awareness that one had a mental illness previously). Our response scale was shortened from 5 to 3 points: for each item, a score of 1 indicated clear awareness, a 2 signified "somewhat aware", and a 3 indicated unawareness. Current and retrospective insight measures were computed by summing the three items for each.

\section{Plan of analysis}

Data were analysed within a causal model framework, using multiple regression to test individual hypotheses. Mediation was tested using the method described by Baron and Kenny (1986). Standardised regression coefficients $(\beta)$ and their associated $t$-statistics are reported.

\section{Interrater reliability}

Cognitive data were gathered while blind to symptom profiles. Nonetheless, to minimise potential bias based on obvious symptoms, interrater reliability was assessed using a second, truly blind, rater. Such evidence was also necessary to demonstrate the reliability of new measures. Interrater reliability for the awareness questionnaire was determined using ratings by both K.C. and R.W.

Pilot data were gathered for the novel cognitive measures, using 12 healthy adults (University of Victoria students and staff), 4 hospitalised, clinically stable patients, and the first 8 study participants $(N=24)$. Scoring techniques were refined and criteria generated, using 11 subjects for the MHP and 16 subjects for the TT. Table 1 presents results for data scored following finalisation of criteria ( $n=13$ for MHP and $n=8$ for TT). Our goal of Pearson $r=.85$ was exceeded for number of words, perseverations, and total inappropriate communications for the MHP. Intermingling and MIRs were not reliable at this time (the majority of the scoring inconsistency occurred in one transcript containing many bizarre communications). All three inhibition errors on the TT, as well as their total, exceeded criterion.

For the final reliability assessment, a stratified random sample $(N=15)$ of the remaining 32 subjects (37.5\% of the total sample) was scored by both raters. Number of words was not tested further given its perfect reliability. In addition, because the number of intermingled ideas had proven unreliable, we added a score for the number of intermingled words (see Table 2). 
TABLE 1

Initial intercorrelations between raters for Picture Description and Tinkertoy test

\begin{tabular}{lc}
\hline Score & $r$ \\
\hline Picture description (MHP; $n=13)$ & \\
Number of words ${ }^{\mathrm{a}}$ & $1.00 * * *$ \\
Intermingling & .28 \\
Missing information references & -.12 \\
Perseverations & $.94 * * *$ \\
Total inappropriate communications & $.89 * * *$ \\
& \\
Tinkertoy test (TT; $n=8)$ & $.89 * *$ \\
False starts & $.97 * * *$ \\
Disconnections & $1.00 * * *$ \\
Odd actions & $.98 * * *$ \\
Total inhibition errors & .66 \\
Appropriateness of name &
\end{tabular}

${ }^{\mathrm{a}} n=10 .{ }^{* *} p<.01 ; * * * p<.001$.

TABLE 2

Test phase intercorrelations between raters for Picture Description and Tinkertoy test

\begin{tabular}{lc}
\hline Score & $r$ \\
\hline Picture Description (MHP; $n=15$ ) & \\
Intermingling (no. of ideas) & $.96^{* * *}$ \\
Intermingling (no. of words) & $.97 * * *$ \\
Missing information references & $.97 * * *$ \\
Perseverations & $.97 * * *$ \\
Total inappropriate communications & $.92^{* * *}$ \\
Tinkertoy test (TT; $n=15$ ) & \\
False starts & $.98^{* * *}$ \\
Disconnections & $.99^{* * *}$ \\
Odd actions ${ }^{\mathrm{a}}$ & - \\
Total inhibition errors & $.99 * * *$ \\
Appropriateness of name & - \\
\hline
\end{tabular}

${ }^{\mathrm{a}}$ Correlation cannot be computed because at least one of the variables is constant. $* * * p<.001$. 
Missing data. MHP latency scores were missing for two participants; the median score for the other 38 subjects was used to estimate these scores. Interrater reliability for the insight questionnaire was based on 36 subjects due to unscorable items or missing data.

\section{RESULTS}

For all statistical tests, the direction of the effect was hypothesised based on theory and previous supporting data from the literature (i.e., neuropsychological performance and cognitive competency were expected to be inversely related to the presence of symptoms). Therefore, one-tailed significance tests were used. To limit the occurrence of Type I errors, a conservative critical $p$-value of .01 was set for all significance tests.

\section{Interrater reliability}

Results are presented in Table 2. All MHP scores revealed high reliabilities (ranging from .92 to .97). For TT odd actions, a reliability coefficient could not be computed because one rater found no occurrences. However, agreement was obtained for $87 \%$ of subjects. Other TT scores showed high reliabilities (.98 and .99). Thus, excellent interrater reliability was demonstrated for the majority of the experimental scores from the unstructured tasks. For insight ratings, interrater reliability was .88 for current and .75 for retrospective awareness.

\section{Cognition, syndromes, and cognitive competency}

The first hypothesis was that weak initiation underlies the psychomotor poverty syndrome. This was tested by a regression of the psychomotor poverty syndrome score on the initiation component. Contrary to expectations, initiation did not predict psychomotor poverty: $t(38)=0.87, p=.20, \beta=.14, S E=0.54$. However, we noted that when only the generative variables within the initiation component were considered (i.e., number of MHP words and number of TT pieces), this relationship bordered on significance in the expected direction: $t(38)=$ $-1.93, p=.03, \beta=-.30, S E=0.33$.

The second hypothesis was that poor inhibition causes the disorganisation syndrome. To test this hypothesis, disorganisation was regressed on the disinhibition aggregate. Disinhibition failed to predict disorganisation: $t(38)=1.17, p$ $=.12, \beta=.19, S E=0.45$. Within the disinhibition aggregate, however, the predictive power of the MHP error ratio bordered on significance: $t(38)=2.22, p$ $=.016, \beta=.34, S E=0.43$, whereas the TT error ratio was clearly not significant: $t(38)=-0.58, p=.72, \beta=-.09, S E=0.46)$. Therefore, we examined more closely the components of the MHP error ratio. Of these, only the intermingling ratio (i.e., the number of intermingled words divided by total number of words) was related to disorganisation: $t(38)=4.36, p<.001, \beta=.58, S E=$ 
0.38. That is, individuals who intermingled more personal material into their stories showed more disorganisation.

The third hypothesis was that both psychomotor poverty and disorganisation predict deficits in cognitive competency. This was tested by regressing cognitive competency on both syndromes simultaneously. Disorganisation was a significant predictor of cognitive competency, in the expected direction: $t(37)=$ $-4.28, p<.001, \beta=-.58, S E=0.48$. That is, participants with more severe disorganisation obtained lower CCT scores. In contrast, psychomotor poverty failed to predict cognitive competency: $t(37)=0.40, p=.65, \beta=.05, S E=0.41$.

As part of the third hypothesis, psychomotor poverty and disorganisation were expected to mediate the impact of initiation and inhibition impairments, respectively, on cognitive competency. Mediation was not possible for initiation/psychomotor poverty, because initiation did not predict psychomotor poverty, and psychomotor poverty did not predict cognitive competency. However, the initiation component was directly related to cognitive competency: $t(38)=-2.59, p<.01, \beta=-.39, S E=1.52$. Thus, patients who produced more speech, incorporated more Tinkertoys, and initiated tasks faster achieved higher CCT scores. Consistent with hypotheses, disorganisation mediated the effect of intermingling (the only relevant inhibition measure) on cognitive competency. That is, although intermingling significantly predicted cognitive competency: $t(38)=-2.51, p<.01, \beta=-.38, S E=1.53$, when disorganisation was entered into the regression equation along with intermingling, the latter no longer significantly predicted cognitive competency: $t(37)=-0.42, p=.34, \beta=-.07, S E=1.67$.

\section{Awareness of illness}

Because awareness of current and of past mental illness were highly intercorrelated $(r=.82, p<.001)$, their sum was used as an overall insight measure. Insight was positively correlated with cognitive competency $(r=.50, p<.001)$; that is, individuals with greater insight had greater cognitive competency. Furthermore, insight's contribution to cognitive competency was unique: $t(36)=$ $3.14, p<.005, \beta=.38, S E=0.30$, over and above the effects of disorganisation and initiation. Of the variables predicting cognitive competency, awareness correlated only with disorganisation $(r=-.41, p<.01)$ : Patients with weaker insight exhibited more disorganisation features.

\section{Predicting cognitive competency}

The variables that predicted cognitive competency, in decreasing order of unique contribution, were: initiation, $t(36)=3.80, p<.001, \beta=.42, S E=1.11$; disorganisation syndrome, $t(36)=-3.40, p<.001, \beta=-.41, S E=0.42$; and insight, $t(37)=3.14, p<.005, \beta=.38, S E=0.30$. Together, initiation, disorganisation, and insight accounted for $58 \%$ of the variance in cognitive com- 


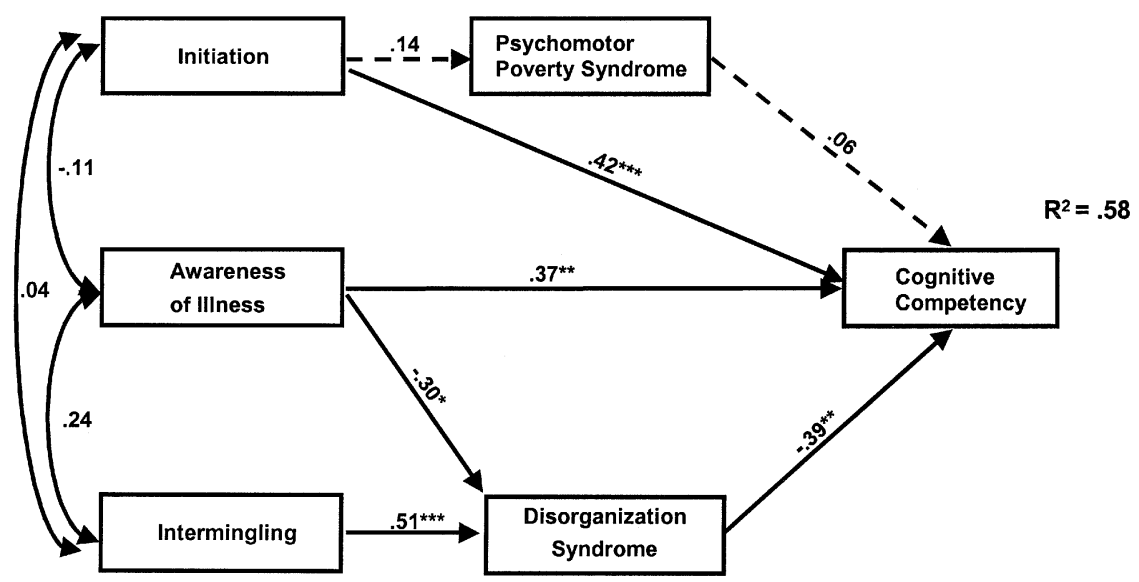

Figure 2. This figure displays the regression results, in the format of a causal model that estimates numerous predictor weights simultaneously. Initiation failed to predict psychomotor poverty, but affected cognitive competency directly and positively. One indicator of disinhibition (intermingling of personal material into speech) predicted disorganisation, which, through mediation, led to cognitive competency deficits. Insight correlated negatively with disorganisation and contributed to cognitive competency. Unique effects of initiation, disorganisation, and insight, combined, explained $58 \%$ of the variance in cognitive competency. $(* p<.05 ; * * p<.01 ; * * *<.001$.)

petency $\left(R^{2}=.58\right)$. Figure 2 displays the results. The coefficients in Figure 2 differ slightly from those reported here, because regressions in such a model include simultaneous estimation of predictor weights for all predictors of each endogenous variable.

\section{Medication effects}

There is evidence that anticholinergic medications can have a negative impact on cognition, especially memory (Tune, Strauss, Lew, Brietlinger, \& Coyle, 1982). These medications were not associated with any cognitive variables in the model, but were negatively related to disorganisation $(r=-.41, p<.01)$. Patients taking anticholinergics exhibited less disorganisation.

\section{DISCUSSION}

The goal of this study was to test a three-level model of schizophrenia pathology (Neuropsychological dysfunction $\rightarrow$ Syndromes $\rightarrow$ Cognitive competency). Cognitive competency represented an important and neglected outcome. Original unstructured tasks were used with the aim of highlighting variability in initiation and inhibition. Findings indicate that specific neuropsychological abilities and symptoms explain $58 \%$ of the variance in cognitive competency. We found some support for the three-level model in that weak inhibition 
capacity - as measured by a tendency to intermingle-predicted the disorganisation syndrome, which in turn led to deficits in cognitive competency. In terms of initiation capacity, a direct impact on cognitive competency was revealed. The exploration of insight within this model confirmed its importance as a unique predictor of cognitive competency, as well as a correlate of the disorganisation syndrome.

Initiation had a direct impact on cognitive competency. A similar observation in neuropsychological testing is that the capacity to make several quick responses to an item enhances the odds of obtaining at least partial scores. The implication is that the ability to generate responses immediately and prolifically increases the likelihood of reacting correctly in real-life situations.

The tendency to allow personal material to disrupt speech (intermingling) was associated with the disorganisation syndrome. To the extent that intermingling measures disinhibition, this result would support the hypothesis that disinhibition underlies disorganisation. It could be argued that intermingling simply indicates disorganisation features (especially tangentiality and derailment, aspects of positive formal thought disorder), rather than reflecting an underlying, causal cognitive deficit. However, intermingling did not correlate with the "thought disorder" aspect of the disorganisation syndrome $(r=.29, p>$ $.01)$, whereas it did correlate with inappropriate affect $(r=.63, p<.001)$, suggesting that intermingling may be a distinct level of pathology.

In addition, intermingling affected cognitive competency through its impact on disorganisation. This portion of the results, although pertaining to only one of the proposed inhibition measures, is consistent with the three-level model. In contrast to our finding of a direct relationship between initiation and cognitive competency, this outcome suggests that the executive deficit explains the functional burdens indirectly, through its effect on the clinical syndrome.

Whether the effect is direct or indirect, these results indicate a prominent role for neuropsychological deficits in determining functional status in schizophrenia. Results are in keeping with previous indications that cognitive skills underlie decision-making capacity and predict functional status in various groups (Green, 1996; Marson, Cody, Ingram, \& Harrell, 1995; Schindler, Ramchandani, Matthews, \& Podell, 1995). In schizophrenia, cognitive deficits have been related to social competence in chronically ill patients (Penn, Mueser, Spaulding, Hope, \& Reed, 1995), and have been considered a rate-limiting factor for acquiring psychosocial skills (Liberman \& Corrigan, 1993). There is previous evidence that measures of executive functioning (e.g., word fluency, the Executive Interview) are superior predictors - compared to other skills including verbal reasoning, memory, and general cognition - of competency outcomes such as rational decision making and level of independence (Marson et al., 1995; Royall et al., 1993).

The unique and significant contribution of insight to cognitive competency is consistent with David's (1998) conclusions that insight represents a distinct 
aspect of psychosis, and is clinically important. Moreover, this finding is expected, given previous research demonstrating that poor insight predicts functional impairments at work (Lysaker \& Bell, 1995), and also predicts the ability to live independently and gainfully employed (van Os et al., 1996). Our result contributes to this literature in documenting that patients who lack awareness of their mental illness tend to experience more difficulty performing relatively simple mental tasks needed for daily living. Clearly, such basic cognitive difficulties as those measured by the CCT would impact functioning in all settings, including work and activities of daily living.

Results indicate that more disorganised patients have less insight. This finding is interesting because the orbitofrontal neural circuit has been implicated in both the disorganisation syndrome (Liddle, Barnes, Morris, \& Haque, 1989; Liddle et al., 1992) and self-awareness (Cicerone \& Tanenbaum, 1997; Cummings, 1993). We found a strong correlation between awareness and disorganisation, whereas Amador and colleagues (Amador et al., 1994) reported fairly weak, but significant, associations between decreased insight and certain disorganisation and reality distortion features (i.e., delusionality, thought disorder, and disorganised behaviour). Others have connected diminished insight with negative symptoms (Amador \& Kronengold, 1998) or described it as separate from the syndromes (Peralta \& Cuesta, 1994). Further clarification is required concerning insight in relation to other schizophrenia symptoms.

The lack of correlation between initiation and psychomotor poverty was unexpected. It contradicts the theory, based on neuropsychological studies, that weak initiation underlies this syndrome. It also challenges the results of Mendez and Ashla-Mendez (1991), who used the same measure of initiation with dementia patients. The present lack of correlation may relate to our sample being less chronically ill (14 patients had been diagnosed for one year or less) compared to previous studies (Allen et al., 1993; Liddle, 1987a; Liddle \& Morris, 1991); less stable symptoms and cognitive deficits could limit correlations. Alternatively, our initiation measure may assess a construct different from the one previously linked to psychomotor poverty (e.g., Liddle \& Morris, 1991). In addition, the study's power may have been insufficient to detect subtle effects; as evidence for this notion, one aspect of initiation (the generative component) was correlated with psychomotor poverty at the trend level. It is interesting to note that, in terms of predicting psychomotor poverty, the ability to maintain response generation over time appears more promising than the ability to initiate a response. Future investigation of this hypothesis would be valuable. A final possibility is that, as Norman et al. (1997) suggest, impaired initiation is not associated with psychomotor poverty.

The other speech features assumed to assess disinhibition were noncontributory in this study. Missing information references were rare; perseverations were unrelated to intermingling and also did not appear unusual. The aggregate of experimental scores from the Tinkertoy test (false starts, dis- 
connections, and odd actions) also failed to relate to disorganisation. (Odd actions was correlated with disorganisation, but only six subjects exhibited odd actions; $r$ $=.39, p<.05$.) In summary, it appears that either: (a) the Tinkertoy test is not helpful in eliciting inhibition impairments; or (b) the scores we derived from the test, with the possible exception of odd actions, are not valid indicators of disinhibition. Specific speech errors appear more promising for this purpose.

A general limitation of this study is the use of unstructured tasks for which reliability and validity data in this population did not exist. Although interpretation of results is challenging at this time, sound rationales support ongoing investigation to enhance the validity of unstructured tasks for studies involving executive functions. For instance, recommendations based on this study include using even more unrestricted verbal tasks (e.g., open-ended questions) to elicit intermingling, and measuring the broader class of "unclear references" (Docherty et al., 1996) rather than only missing information references. Prior to future use of these and other measures from unstructured tasks, their validity should be examined, using a multitrait-multimethod matrix (Campbell \& Fiske, 1959). This methodology, based on measurement theory, asserts that a test aiming to measure a particular attribute should correlate highly with other measures of the same attribute (convergent validity), while demonstrating lower correlations with tests of different attributes (discriminant validity). Prior studies have used this approach as a relatively stringent test of construct validity (e.g., Christensen \& Joschko, 2001). To test whether intermingling is a specific measure of disinhibition, for example, one would expect correlations of intermingling with established measures of disinhibition (e.g., Stroop interference; WCST perseverations), while hypothesising nonsignificant correlations with unrelated skills and potential confounds (e.g., general intellectual ability; language skills). Another general limitation is that our sample size was relatively small, and Type II errors may have occurred due to insufficient power.

This study provides only partial support for our unique three-level model of schizophrenia pathology (Neuropsychological dysfunction $\rightarrow$ Syndromes $\rightarrow$ Cognitive competency). Additional research is required to determine whether the proposed causal pathways are upheld. More encouraging is the fact that $58 \%$ of the variance in cognitive competency is explained by the combination of initiation capacity, disorganisation syndrome, and level of insight. The implication is that if a person's functioning in these domains were maximised, a significant improvement in cognitive competency may result. Such changes could potentially reduce future hospitalisations, use of assistance/supervision resources to support the patient outside the hospital, and the disruption of family life. These results and others to come may prove useful in directing treatment and remediation efforts for maximal benefit and cost-effectiveness. 


\section{REFERENCES}

Allen, H. A., Liddle, P. F., \& Frith, C. D. (1993). Negative features, retrieval processes and verbal fluency in schizophrenia. British Journal of Psychiatry, 163, 769-775.

Amador, X. F., Flaum, M., Andreasen, N. C., Strauss, D. H., Yale, S. A., Clark, S. C., \& Gorman, J. M. (1994). Awareness of illness in schizophrenia and schizoaffective and mood disorders. Archives of General Psychiatry, 51, 826-836.

Amador, X. F., \& Kronengold, H. (1998). The description and meaning of insight in psychosis. In X. F. Amador \& H. Kronengold (Eds.), Insight and psychosis (pp. 15-32). New York: Oxford University Press.

Amador, X. F., \& Strauss, D. H. (1990). The scale to assess unawareness of mental disorder (SUMD). Columbia University and New York State Psychiatric Institute.

Amador, X. F., Strauss, D. H., Yale, S. A., Flaum, M. M., Endicott, J., \& Gorman, J. M. (1993). Assessment of insight in psychosis. American Journal of Psychiatry, 150, 873-879.

Amador, X. F., Strauss, D. H., Yale, S. A., \& Gorman, J. M. (1991). Awareness of illness in schizophrenia. Schizophrenia Bulletin, 17, 113-132.

American Psychiatric Association. (1994). Diagnostic and statistical manual of mental disorders (4th ed.). Washington, DC: Author.

Ammons, R. B., \& Ammons, C. H. (1962). Monograph Supplement I-VII. Psychological Reports. Missoula, MT: Psychological Test Specialists.

Andreasen, N. C. (1983). The scale for the assessment of negative symptoms (SANS). Iowa City, IA: University of Iowa Press.

Andreasen, N. C. (1984). The scale for the assessment of positive symptoms (SAPS). Iowa City, IA: University of Iowa Press.

Baron, R. M., \& Kenny, D. A. (1986). The moderator-mediator variable distinction in social psychological research: Conceptual, strategic, and statistical considerations. Journal of Personality and Social Psychology, 51, 1173-1182.

Bayless, J. D., Varney, N. R., \& Roberts, R. J. (1989). Tinker Toy Test performance and vocational outcome in patients with closed-head injuries. Journal of Clinical and Experimental Neuropsychology, 11, 913-917.

Blumer, D., \& Benson, D. F. (1975). Personality changes with frontal and temporal lobe lesion. In D. F. Benson \& D. Blumer (Eds.), Psychiatric aspects of neurologic disease (pp. 151-170). New York: Grune \& Stratton.

Campbell, D. T., \& Fiske, D. W. (1959). Convergent and discriminant validation by the multitraitmultimethod matrix. Psychological Bulletin, 56, 81-105.

Christensen, K. M. (1998). Initiation and inhibition deficits, syndromes, and cognitive competency in schizophrenia. University of Victoria, Victoria, BC, Canada.

Christensen, K. M., \& Joschko, M. (2001). Construct validity of the Continuous Attention Test for Children. The Clinical Neuropsychologist, 15, 203-209.

Cicerone, K. D., \& Tanenbaum, L. N. (1997). Disturbance of social cognition after traumatic orbitofrontal brain injury. Archives of Clinical Neuropsychology, 12, 173-188.

Cummings, J. L. (1993). Frontal-subcortical circuits and human behavior. Archives of Neurology, 50, 873-880.

David, A. S. (1998). The clinical importance of insight. In X. F. Amador \& A. S. David (Eds.), Insight and psychosis (pp. 332-351). New York: Oxford University Press.

Docherty, N. M., DeRosa, M., \& Andreasen, N. C. (1996). Communication disturbances in schizophrenia and mania. Archives of General Psychiatry, 53, 358-364.

Frith, C. D. (1992). The cognitive neuropsychology of schizophrenia. Hove, UK: Psychology Press.

Frith, C. D., \& Done, D. J. (1988). Towards a neuropsychology of schizophrenia. British Journal of Psychiatry, 153, 437-443.

Frith, C. D., \& Done, D. J. (1989). Experiences of alien control in schizophrenia reflect a disorder in the central monitoring of action. Psychological Medicine, 19, 359-363. 
Frith, C. D., Leary, J., Cahill, C., \& Johnstone, E. C. (1991). Disabilities and circumstances of schizophrenic patients - a follow-up study: IV. Performance on psychological tests. British Journal of Psychiatry, 159(Suppl. 13), 26-29.

Green, M. F. (1996). What are the functional consequences of neurocognitive deficits in schizophrenia? American Journal of Psychiatry, 153, 321-330.

Green, M. F. (1998). Schizophrenia from a neurocognitive perspective: Probing the impenetrable darkness. Needham Heights, MA: Allyn \& Bacon.

Hammer, M. A., Katsanis, J., \& Iacono, W. G. (1995). The relationship between negative symptoms and neuropsychological performance. Biological Psychiatry, 37, 828-830.

Hammill, D. D., \& Larsen, S. C. (1988). Test of written language (2nd ed.). Austin, TX: Pro-Ed.

Harrow, M., Lanin-Kettering, I., Prosen, M., \& Miller, J. G. (1983). Disordered thinking in schizophrenia: Intermingling and loss of set. Schizophrenia Bulletin, 9, 354-367.

Harrow, M., \& Prosen, M. (1979). Schizophrenic thought disorder: Bizarre associations and intermingling. American Journal of Psychiatry, 136, 293-296.

Hauser, M. D. (1999). Perseveration, inhibition and the prefrontal cortex: A new look. Current Opinion in Neurobiology, 9, 214-22.

Lezak, M. D. (1982). The problem of assessing executive functions. International Journal of Psychology, 17, 281-297.

Liberman, R. P., \& Corrigan, P. W. (1993). Designing new psychosocial treatments for schizophrenia. Psychiatry, 56, 238-249.

Liddle, P. F. (1987a). Schizophrenic syndromes, cognitive performance and neurological dysfunction. Psychological Medicine, 17, 49-57.

Liddle, P. F. (1987b). The symptoms of chronic schizophrenia: A re-examination of the positivenegative dichotomy. British Journal of Psychiatry, 151, 145-151.

Liddle, P. F. (2001). Disordered mind and brain: The neural basis of mental symptoms. London: Gaskell.

Liddle, P. F., \& Barnes, T. R. E. (1990). Syndromes of chronic schizophrenia. British Journal of Psychiatry, 157, 558-561.

Liddle, P. F., Barnes, T. R. E., Morris, D., \& Haque, S. (1989). Three syndromes in chronic schizophrenia. British Journal of Psychiatry, 155(Suppl. 7), 119-122.

Liddle, P. F., Friston, K. J., Frith, C. D., Hirsch, S. R., Jones, T., \& Frackowiak, R. S. (1992). Patterns of cerebral blood flow in schizophrenia. British Journal of Psychiatry, 160, 179-186.

Liddle, P. F., \& Morris, D. L. (1991). Schizophrenic syndromes and frontal lobe performance. British Journal of Psychiatry, 158, 340-345.

Lysaker, P., \& Bell, M. (1994). Insight and cognitive impairment in schizophrenia: Performance on repeated administrations of the Wisconsin Card Sorting Test. Journal of Nervous and Mental Disease, 182, 656-660.

Lysaker, P., \& Bell, M. (1995). Work rehabilitation and improvements in insight in schizophrenia. Journal of Nervous and Mental Disease, 183, 103-106.

Malla, A. K., Norman, R. M. G., Williamson, P., Cortese, L., \& Diaz, F. (1993). Three syndrome concept of schizophrenia: A factor analytic study. Schizophrenia Research, 10, 143150 .

Marson, D. C., Cody, H. A., Ingram, K. K., \& Harrell, L. E. (1995). Neuropsychological predictors of competency in Alzheimer's disease using a rational reasons legal standard. Archives of Neurology, 52, 955-959.

Martzke, J. S., Swan, C. S., \& Varney, N. R. (1991). Posttraumatic anosmia and orbital frontal damage: Neuropsychological and neuropsychiatric correlates. Neuropsychology, 5, 213-225.

Mendez, M. F., \& Ashla-Mendez, M. (1991). Differences between multi-infarct dementia and Alzheimer's disease on unstructured neuropsychological tasks. Journal of Clinical and Experimental Neuropsychology, 13, 923-932.

Mlakar, J., Jensterle, J., \& Frith, C. D. (1994). Central monitoring deficiency and schizophrenic symptoms. Psychological Medicine, 24, 557-564. 
Moritz, S., Andresen, B., Jacobsen, D., Mersmann, K., Wilke, U., Lambert, M., Krausz, M., \& Naber, D. (2001). Neuropsychological correlates of schizophrenic syndromes in patients treated with atypical neuroleptics. European Psychiatry, 16, 354-361.

Mortimer, A. (1994). Levels of explanation-symptoms, neuropsychological deficit and morphological abnormalities in schizophrenia. Psychological Medicine, 24, 541-545.

Mortimer, A. M. (1992). Phenomenology: Its place in schizophrenia research. British Journal of Psychiatry, 161, 293-297.

Norman, R. M. G., Malla, A. K., Morrison-Stewart, S. L., Helmes, E., Williamson, P. C., Thomas, J., \& Cortese, L. (1997). Neuropsychological correlates of syndromes in schizophrenia. British Journal of Psychiatry, 170, 134-139.

Penn, D. L., Mueser, K. T., Spaulding, W., Hope, D. A., \& Reed, D. (1995). Information processing and social competence in chronic schizophrenia. Schizophrenia Bulletin, 21, 269-281.

Peralta, V., \& Cuesta, M. J. (1994). Lack of insight: Its status within schizophrenic psychopathology. Biological Psychiatry, 36, 559-561.

Royall, D. R., Mahurin, R. K., True, J. E., Anderson, B., Brock, I. P., et al. (1993). Executive impairment among the functionally dependent: Comparisons between schizophrenic and elderly subjects. American Journal of Psychiatry, 150, 1813-1819.

Schindler, B. A., Ramchandani, D., Matthews, M. K., \& Podell, K. (1995). Competency and the frontal lobe: The impact of executive dysfunction on decisional capacity. Psychosomatics, 36, 400-404.

Tune, L. E., Strauss, M. E., Lew, M. F., Brietlinger, E., \& Coyle, J. T. (1982). Serum levels of anticholinergic drugs and impaired recent memory in chronic schizophrenic patients. American Journal of Psychiatry, 139, 1460-1462.

van Os, J., Fahy, T., Jones, P., Harvey, I., Sham, P., Lewis, S., Toone, B., Williams, M., \& Murray, R. (1996). Psychopathological syndromes in the functional psychoses: Associations with course and outcome. Psychological Medicine, 26, 161-176.

Wang, P. L. (1990). Assessment of cognitive competency. In D. E. Tupper \& K. D. Cicerone (Eds.), The neuropsychology of everyday life: Assessment and basic competencies. Norwell, MA: Kluwer.

Wang, P. L., Ennis, K., \& Copland, S. (1987). Cognitive Competency Test. Toronto: Mount Sinai Hospital, Psychology Department.

Wang, P. L., \& Ennis, K. E. (1986). Competency assessment in clinical populations: An introduction to the Cognitive Competency Test. In B. Uzzell \& Y. Gross (Eds.), Clinical neuropsychology of intervention (pp. 199-133). Boston: Martinus Nijhoff.

Woodward, T. S., Ruff, C. C., Thornton, A. E., Moritz, S., \& Liddle, P. (2003). Methodological considerations regarding the association of Stroop and verbal fluency performance with the symptoms of schizophrenia. Schizophrenia Research, 61, 207-214.

Woodward, T. S., Thornton, A. E., Ruff, C. C., Moritz, S., \& Liddle, P. (2004). Material-specific memory associates of the psychomotor poverty syndrome in schizophrenia. Cognitive Neuropsychiatry, 9, 213-227.

Young, D. A., Davila, R., \& Scher, H. (1993). Unawareness of illness and neuropsychological performance in chronic schizophrenia. Schizophrenia Research, 10, 117-124. 
Copyright of Cognitive Neuropsychiatry is the property of Psychology Press (UK). The copyright in an individual article may be maintained by the author in certain cases. Content may not be copied or emailed to multiple sites or posted to a listserv without the copyright holder's express written permission. However, users may print, download, or email articles for individual use. 